\title{
COVID-19 among Pregnant Women Delivering in a Tertiary Care Center: A Descriptive Cross-sectional Study
}

\author{
Pooja Paudyal,' ${ }^{1}$ Neeta Katuwal, ' Suniti Rawal' \\ 'Department of Obstetrics and Gynecology, Tribhuvan University Teaching Hospital, Maharajgunj Medical \\ Campus, Institute of Medicine, Kathmandu, Nepal.
}

\begin{abstract}
Introduction: Coronavirus Disease 2019 pandemic is raging across the world and has affected pregnant women as well. There is limited information regarding COVID-19 in pregnant women. The study aimed to find the prevalence of COVID-19 among all pregnant women who delivered during the study period in a tertiary care center.
\end{abstract}

Methods: This was a descriptive cross-sectional study conducted in a tertiary care center from $16^{\text {th }}$ August to $15^{\text {th }}$ November 2020 after obtaining ethical clearance from the Institutional Review Committee of a tertiary care center. All the women who delivered in the hospital during the study period were enrolled and they were subjected to COVID-19 Reverse Transcriptase Polymerase Chain Reaction test. A total of 667 samples were taken using convenience sampling technique. Data were analyzed using the Statistical Package for the Social Sciences version 24 software. Point estimate at 95\% Confidence Interval was calculated along with frequency and proportion for binary data.

Results: Among 667 pregnant women, the prevalence of COVID-19 was 47 (7.05\%) (5.10-8.99 at 95\% Confidence Interval). Though the majority of women were asymptomatic $40(85.1 \%), 5(10.64 \%)$ developed mild disease, 1 (2.12\%) each had severe and critical COVID-19 pneumonia.

Conclusions: The prevalence of COVID-19 among pregnant women delivering in our center is similar to other studies done in similar settings. In our study, we found that the majority of women had been asymptomatic and were diagnosed on routine testing. Hence, it is important to test all pregnant women before delivery for Coronavirus Disease 2019 irrespective of the presence or absence of symptoms.

Keywords: COVID-19; obstetric delivery; pneumonia; pregnancy; reverse transcriptase PCR.

\section{INTRODUCTION}

Coronavirus Disease 2019 (COVID-19); caused by severe acute respiratory syndrome coronavirus 2 (SARS-CoV-2) first emerged in Wuhan, Hubei Province, China in November 2019 and was declared a global pandemic in March 2020 by World Health Organization (WHO); has brought the world down to its heels. ${ }^{1-4}$ The pandemic has affected each one of us and has brought our lives to a standstill. Many have lost their dear and near ones to this unforgiving pandemic.

Pregnant women have not been spared either. The effects of this disease in pregnancy are still under study and information regarding the epidemiology and effects of COVID-19 in pregnancy is limited more so in a country like Nepal.

The aim of this study was to find the prevalence of COVID-19 among all pregnant women who delivered during the study period in a tertiary care center.

\section{METHODS}

This was a descriptive cross-sectional study conducted in the Department of Obstetrics and Gynecology of a tertiary care center. The duration of the study was 3

Correspondence: Dr. Pooja Paudyal, Tribhuvan University Teaching Hospital, Maharajgunj Medical Campus, Institute of Medicine, Kathmandu, Nepal. Email: paudyalpooja@yahoo.com, Phone: +977- 9841526853 . 
months from $16^{\text {th }}$ August to $15^{\text {th }}$ November 2020. Ethical clearance was obtained from the Institutional Review Committee of the Institute of Medicine. All women who delivered during the study period and who had had the COVID-19 tests were studied. It was hospital protocol to subject all women admitted for delivery in the center to COVID-19 Reverse Transcriptase Polymerase Chain Reaction (RT- PCR) test and all the women who delivered in the hospital during the study period were enrolled. All patients diagnosed with COVID-19 via a nasopharyngeal swab were analyzed. A convenience sampling technique was used.

The sample size was calculated using the following formula:

$$
\begin{aligned}
\mathrm{n} & =Z^{2} \times(\mathrm{p} \times \mathrm{q}) / \mathrm{e}^{2} \\
& =(1.96)^{2} \times 0.5 \times(1-0.5) /(0.04)^{2} \\
& =601
\end{aligned}
$$

Where,

$\mathrm{n}=$ minimum required sample size

$\mathrm{Z}=\mathbf{1 . 9 6}$ at $95 \%$ Confidence Interval (Cl)

$\mathrm{p}=$ prevalence of COVID-19 among all pregnant women who delivered during the study period in a tertiary care center taken as $50 \%$ for maximum sample size calculation

$q=1-p$

$e=$ margin of error, $4 \%$

Adding a non-response rate of $10 \%$, the final sample size was 662 . However, a total of 667 samples were taken.

Our study participants were the pregnant women with laboratory-confirmed SARS-CoV-2 infection on an RT-PCR assay performed on a nasopharyngeal swab before or during delivery hospitalization. Data were collected from patient files regarding age, parity, occupation, and travel history, history of exposure, symptoms, and treatments received if any were also recorded. Other variables studied were comorbidities associated, mode of delivery, maternal and perinatal outcomes.

The criteria for classifying COVID-19 positive patients into severity grades are as follows:

1. Asymptomatic: Those who reported being in usual health without signs or symptoms of COVID-19.3,4

2. Mild COVID-19: Symptoms requiring no additional oxygen supplementation or treatment beyond standard labor and delivery care..$^{3,4}$

3. Severe COVID-19: Dyspnea, respiratory rate 30 breaths per minute or higher, oxygen saturation $93 \%$ or less on room air, or findings consistent with pneumonia on chest X-ray, or a combination of these..$^{3,4}$

4. Critical COVID-19- any or all of the following: respiratory failure (need for intubation and invasive ventilation), septic shock, and multiple organ dysfunction or failure..$^{3,4}$

Data were analyzed using the Statistical Package for the Social Sciences version 24 (SPSS) software and results were presented in appropriate tables. Point estimate at $95 \%$ Confidence Interval was calculated along with frequency, proportion, mean and standard deviations for binary data.

\section{RESULTS}

Among 667 women, who delivered in our hospital during the study period, the prevalence of Coronavirus Disease 2019 was 47 (7.05\%) (5.10-8.99 at 95\% Confidence Interval). The mean age of the COVID-19 positive women was $29 \pm 4.438$ years varying from a minimum of 20 years to a maximum of 35 years, and the majority 27 (57.4\%) were multigravida. Regarding occupation, most were homemakers 36 (76.60\%), followed by five health care workers (nurse 4, doctor 1) (Table 1).

\begin{tabular}{|lcl|}
\hline \multicolumn{3}{|l|}{ Table 1. Maternal characteristics $(\mathbf{n}=47)$} \\
\hline Parameters & $\mathbf{n}(\%)$ & Mean \pm SD \\
Age (in years) & & $29 \pm 4.438$ \\
$20-24$ & $9(19)$ & \\
$25-30$ & $15(32)$ & \\
$>30$ & $23(49)$ & \\
Parity & & $1.57 \pm 0.5$ \\
Primigravida & $20(42.5)$ \\
Multigravida & $27(57.4)$ \\
Occupation & \\
Homemakers & $36(76.6)$ \\
Health care workers & $5(10.6)$ \\
Business owners & $2(4.26)$ \\
Shopkeepers & $2(4.26)$ \\
Service & $1(2.13)$ \\
Teachers & $1(2.13)$ \\
\hline
\end{tabular}

The average duration of hospital stay was $4.89 \pm 3.661$ days (minimum $=1$ day; maximum $=17$ days).

Of the 47 women; only 5 (10.64\%) had symptoms when they were tested, the rest 42 (89.36\%) were diagnosed on screening at the time of admission for delivery; while 2 (4.2\%) developed symptoms postdelivery. Similarly, 5 (10.6\%) had a history of exposure (one exposed from husband, one from a coworker, and three others were health care workers) and only three $(6.38 \%)$ reported travel history, rest 44 (93.62\%) COVID-19 positive pregnant women reported no travel history (Table 2). 
Paudyal et al. COVID-19 among Pregnant Women Delivering in a Tertiary Care Center: A Descriptive Cross-sectional Study

\begin{tabular}{|ll|}
\hline $\begin{array}{l}\text { Table 2. Symptoms, exposure, and travel history } \\
(\mathbf{n}=47) .\end{array}$ \\
\hline Parameters & $\mathbf{n}(\%)$ \\
\hline Asymptomatic & $40(85.1)$ \\
Symptomatic & $7(14.9)$ \\
Fever & $4(8.51)$ \\
Fever + cough & $1(2.12)$ \\
Fever + cough + shortness of breath & $2(4.25)$ \\
History of exposure & \\
Yes & $5(10.6)$ \\
No & $42(89.4)$ \\
History of travel & \\
Yes & $3(6.38)$ \\
No & $44(93.6)$ \\
\hline
\end{tabular}

Twenty-three (49\%) had some associated comorbidity, most commonly hypertension in 8 (34.7\%) and diabetes in $5(21.7 \%)$. Others were hypothyroidism 5 $(21.7 \%)$, Rheumatic Heart Disease 2 (8.7\%), obesity 2 $(8.7 \%)$, obstetric cholestasis $1(4.3 \%)$, Takayasu arteritis $1(4.3 \%)$ and thrombocytopenia 1 (4.3\%).

Though the majority $40(85.1 \%)$ of the women were asymptomatic, $5(10.6 \%)$ developed the mild disease, $1(2.12 \%)$ had severe disease and $1(2.12 \%)$ developed critical COVID pneumonia. All patients recovered and there was no mortality (Table 3 ).

\begin{tabular}{|ll|}
\hline Table 3. Maternal outcome $(\mathbf{n}=\mathbf{4 7})$. & \\
\hline Parameters & $\mathbf{n}(\%)$ \\
Morbidity & \\
Mild disease & $5(10.6)$ \\
Severe COVID disease & $1(2.12)$ \\
Critical COVID disease & $1(2.12)$ \\
Interventions in severe/critical disease & \\
ICU/HDU* admission & $2(4.25)$ \\
Non-invasive ventilation & $1(2.12)$ \\
Invasive mechanical ventilation & $1(2.12)$ \\
Remdesivir & $2(4.25)$ \\
Convalescent Plasma Therapy & $1(2.12)$ \\
Mortality & - \\
\hline
\end{tabular}

*ICU-Intensive Care Unit, HDU-High Dependency Unit

The mean gestational age at COVID-19 diagnosis was $37.83 \pm 2.24$ weeks (minimum = 28.57 weeks; maximum $=40.71$ weeks). The mean age of gestation at delivery was $38.38 \pm 2.24$ weeks (minimum $=28.71$ weeks; maximum = 41 weeks). There were $8(17 \%)$ preterm births and one twin set. The mode of delivery was decided as per obstetric indications and only one patient underwent emergency cesarean Section (CS) at 37 weeks for worsening critical COVID19 pneumonia. Out of 47 , only $4(8.5 \%)$ delivered vaginally, rest 43 $(91.5 \%)$ had CS births. Eleven (23.4\%) had elective CS and $32(68.08 \%)$ had emergency surgery (Table 4$)$.

\begin{tabular}{|ll|}
\hline Table 4. Delivery details $(\mathbf{n}=\mathbf{4 7})$. \\
\hline Parameters & $\mathbf{n}(\%)$ \\
Term & $39(83)$ \\
Preterm & $8(17)$ \\
$28-32$ weeks & $2(4.25)$ \\
$34-36$ weeks & $2(4.25)$ \\
$36-36^{+6}$ weeks & $4(8.51)$ \\
Mode of delivery & \\
Vaginal Delivery & $4(8.5)$ \\
Cesarean Section & $43(91.5)$ \\
Elective Cesarean Section & $11(23.40)$ \\
Emergency Cesarean Section & $32(68.08)$ \\
\hline
\end{tabular}

There were 48 babies born to the 47 COVID-19 positive women including one twin set. There were no intrauterine fetal deaths (IUFD), neonatal deaths (NND), or stillbirths. There were $9(18.7 \%)$ babies with low birthweight. Three babies (6.25) had low APGAR scores at one minute while $2(4.17 \%)$ of them had improved APGAR at five minutes; one baby born to the mother with critical COVID-19, still had low APGAR even at five minutes. The baby was diagnosed to have perinatal depression and was admitted to the Neonatal Intensive Care Unit (NICU), intubated and mechanically ventilated but made a good recovery. There were two others needing admission to NICU for Respiratory Distress Syndrome (RDS) and Meconium Aspiration Syndrome (MAS) while three (6.25\%) babies were admitted to Neonatal Unit (NNU) for transient tachypnea of the newborn (TTN), RDS, and supportive care for the preterm baby born at 28 weeks (Table 5).

\begin{tabular}{|c|c|}
\hline Parameters & n (\%) \\
\hline Low Birth Weight & $9(18.7)$ \\
\hline$<1000 \mathrm{gm}$ & $1(2.12)$ \\
\hline $1000-1500 \mathrm{gm}$ & $1(2.12)$ \\
\hline $1500-2000 \mathrm{gm}$ & $1(2.12)$ \\
\hline $2000-2500 \mathrm{gm}$ & $6(12.76)$ \\
\hline Low APGAR score & $4(33.33)$ \\
\hline 1 minute & $3(6.25)$ \\
\hline 5 minutes & $1(2.12)$ \\
\hline NNU admission & $3(6.25)$ \\
\hline Transient tachypnea of the newborn & $1(33.33)$ \\
\hline Respiratory Distress Syndrome & $1(33.33)$ \\
\hline $\begin{array}{l}\text { Supportive for Intrauterine Growth } \\
\text { Restriction }\end{array}$ & $1(33.33)$ \\
\hline NICU admission & $3(6.25)$ \\
\hline Respiratory Distress Syndrome & $1(33.33)$ \\
\hline Meconium Aspiration Syndrome & $1(33.33)$ \\
\hline Perinatal depression & $1(33.33)$ \\
\hline Intrauterine Fetal Death /Stillbirth & - \\
\hline Neonatal Death & - \\
\hline
\end{tabular}




\section{DISCUSSION}

The effects of COVID-19 during pregnancy are a burning concern as the disease is showing no signs of abating any time soon. Literature on COVID-19 in pregnancy is evolving each day- some have shown poor outcomes, some have reported favorable outcomes while others found no definite difference in COVID-19 course in pregnant as compared to non-pregnant subjects. ${ }^{5-9}$

Most of the women in the present study were asymptomatic $(85.1 \%)$ and were diagnosed on screening at admission at the time of delivery. Fever was the commonest symptom, with all 7 (14.9\%) symptomatic patients having fever. Chen, et al. and Nambiar, et al. reported similar results; most patients were asymptomatic and the commonest symptoms were fever, cough, and fatigue. ${ }^{6,7}$ Majority had no travel or exposure history pointing towards community spread of the disease, similar to study in South India by Nambiar, et al. and Shah, et al. from Gujrat. ${ }^{6,10}$

Five women (10.6\%) had mild disease, 1 (2.12\%) developed the severe disease, and another 1 (2.12\%) developed the critical disease. Guan, et al, in a study from China reported a severe disease rate of $8 \%$ in their pregnant population as compared to $15.7 \%$ risk of severe disease in the general population. ${ }^{5}$ Shah, et al. also reported that only 7 of 125 (5.6\%) pregnant women experienced severe COVID-19 infection. ${ }^{10}$ It is postulated that relative suppression of cell-mediated immunity in pregnancy may be responsible for milder symptoms in COVID-19-positive pregnancies though adequate evidence is not available to conclude the definite effect of this virus in pregnancy. ${ }^{11}$ However, Khoury, et al. have reported a higher rate of severe disease in $16.2 \% .^{12}$

Some studies showed increased rates of preterm labor. ${ }^{13,14}$ Khoury, et al. reported $14.6 \%$ preterm births whereas Chen, et al. reported that four of the nine patients had preterm delivery but the preterm delivery was not associated with COVID-19 pneumonia.,12 In our study majority delivered at term with only 8 (17\%) preterm births and those were unrelated to COVID-19.

The high Cesarean Section rate of $91.5 \%$ in COVID-19 positive patients in our study is double the institutional rate of $45 \%$ during the same period for non- COVID-19 patients. In a study by Nambiar, et al. there was a similarly disproportionate increase in the rate of cesarean sections $(52.28 \%)$ when compared to an institutional rate of $23 \%$ in non-COVID-19 pregnancies. $^{6}$
The delivery policy for COVID-19 patients in our hospital was as per the Royal College Of Obstetricians and Gynecologists (RCOG), which recommends that the mode of delivery be determined primarily by obstetric indications. ${ }^{15}$ The high CS rates could be attributed to a higher proportion of high-risk pregnancies with associated comorbidities in our study and an increased number of repeat Cesarean Sections.

All the newborns did well in our study. None of the babies developed any symptoms of COVID-19, similar to the study by Nambiar, et al. ${ }^{6}$ The babies were not tested for COVID-19 as the protocol was to test babies only if they became symptomatic. Mothers were allowed to room in with the babies and allowed to breastfeed with strict hand wash instructions and wearing a mask every time. This was in accordance with the RCOG, which recommends against the routine separation of COVID-19-affected mothers and their babies, and WHO guidelines, which states that 'the benefits of skin-to-skin contact and breastfeeding substantially outweigh the potential risks of transmission and illness associated with COVID-19 infection'. ${ }^{15-16}$ Only the babies of the two sick mothers were put in the care of their family members till their mothers were in ICU.

The limitations of the study were the small sample size, short duration, and single center. Neonates were not tested for COVID-19; hence we failed to shed any light on the chances of vertical transmission.

\section{CONCLUSIONS}

The prevalence of COVID-19 among pregnant women giving birth in our study is similar to other studies done in similar settings. In our study, we found that the majority of women had been asymptomatic and were diagnosed on routine testing. Of the symptomatic ones, most had only mild diseases that required no special treatment, however, we must watch out for severe cases since they may deteriorate rapidly. Our study stresses the importance of testing all pregnant women before delivery for COVID-19 irrespective of the presence or absence of symptoms.

\section{ACKNOWLEDGEMENTS}

We would like to acknowledge the faculty and residents of the Department of Obstetrics and Gynecology and Dr. Sujaya Rauniyar for their help.

\section{Conflict of Interest: None.}

[cited 2021 Aug 6]. Available from: https://www.who.int/ emergencies/diseases/novel-coronavirus-2019. [․ull Text]

2. Zhu N, Zhang D, Wang W, Li X, Yang B, Song J, et al. A

\section{REFERENCES}

1. World Health Organization. Coronavirus disease(COVID-19) pandemic [Internet]. Geneva: WHO publications; 2020 
Novel Coronavirus from Patients with Pneumonia in China, 2019. N Engl J Med. 2020 Feb 20;382(8):727-33. [PubMed | Full Text | DOI]

3. World Health Organization. Report of the WHO-China Joint Mission on Coronavirus Disease 2019 (COVID-19) [Internet]. Geneva: World Health Organization; 2020 Feb 28 [cited 2021 Aug 6]. Available from: https://www.who.int/ publications/i/item/report-of-the-who-china-joint-mission-on-coronavirus-disease-2019-(covid-19). [Full Text]

4. Wu JT, Leung K, Bushman M, Kishore N, Niehus R, de Salazar PM, et al. Estimating clinical severity of COVID-19 from the transmission dynamics in Wuhan, China. Nat Med. 2020 Apr;26(4):506-10. [PubMed | Full Text | DOI]

5. Guan WJ, Ni ZY, Hu Y, Liang WH, Ou CQ, He JX, et al. Clinical Characteristics of Coronavirus Disease 2019 in China. N Engl J Med. 2020 Apr 30;382(18):1708-20. [PubMed | Full Text | DOI]

6. Nambiar SS, Ajith S, Reshmi V. Assessing Disease Outcome in COVID-19 Pregnancies in a Tertiary Referral Center in South India: A Single-center Retrospective Cohort Study. Journal of SAFOG. 2020 Sep-Oct;12(5):335-9. [Full Text | DOI]

7. Chen H, Guo J, Wang C, Luo F, Yu X, Zhang W, et al. Clinical characteristics and intrauterine vertical transmission potential of COVID-19 infection in nine pregnant women: a retrospective review of medical records. Lancet. 2020 Mar 7;395(10226):809-15. [PubMed | Full Text | DOI]

8. Liu H, Liu F, Li J, Zhang T, Wang D, Lan W. Clinical and CT imaging features of the COVID-19 pneumonia: Focus on pregnant women and children. J Infect. 2020

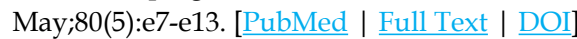

9. Ferrazzi E, Frigerio L, Savasi V, Vergani P, Prefumo F, Barresi S, et al. Vaginal delivery in SARS-CoV-2-infected pregnant women in Northern Italy: a retrospective analysis. BJOG. 2020 Aug;127(9):1116-21. [PubMed | Full Text | DOI]

10. Shah PT, Shah SR, Shah SR, Yadav PA, Patel BS, Chudasama TJ. Fetomaternal outcome in COVID-19 infected pregnant women: a preliminary clinical study. Int J Reprod Contracept Obstet Gynecol. 2020 Sep;9(9):3704-10. [Full Text | DOI]
11. Royal College of Obstetricians and Gynaecologists. Coronavirus (COVID-19) Infection in Pregnancy [Internet]. United Kingdom: Royal College of Obstetricians and Gynaecologists; 2020 [cited 2020 Apr 29]. Available from: https://www.rcog.org.uk/globalassets/documents/ guidelines / 2020-07-24-coronavirus-covid-19-infectionin-pregnancy.pdf. [Full Text]

12. Khoury R, Bernstein PS, Debolt C, Stone J, Sutton DM, Simpson LL, et al. Characteristics and Outcomes of 241 Births to Women With Severe Acute Respiratory Syndrome Coronavirus 2 (SARS-CoV-2) Infection at Five New York City Medical Centers. Obstet Gynecol. 2020 Aug;136(2):273-82. [PubMed | Full Text | DOI]

13. Zhu H, Wang L, Fang C, Peng S, Zhang L, Chang G, et al. Clinical analysis of 10 neonates born to mothers with 2019-nCoV pneumonia. Transl Pediatr. 2020 Feb;9(1):51-60. [PubMed | Full Text | DOI]

14. Yang $\mathrm{R}$, Mei $\mathrm{H}$, Zheng $\mathrm{T}$, Fu Q, Zhang $\mathrm{Y}$, Buka S, et al. Pregnant women with COVID-19 and risk of adverse birth outcomes and maternal-fetal vertical transmission: a population-based cohort study in Wuhan, China. BMC Med. 2020 Oct 19;18(1):330. [PubMed | Full Text | DOI]

15. The Royal College of Obstetricians Gynaecologists. Coronavirus (Covid-19) infection in pregnancy: information for healthcare professionals [Internet]. United Kingdom: Royal College of Obstetricians and Gynaecologists; 2020 Apr 9 [cited 2020 Apr 13]. Available from: https://www. networks.nhs.uk/news/coronavirus-covid-19-infection-in-pregnancy-information-for-healthcare-professionals. [Full Text]

16. World Health Organization. Frequently asked questions: breastfeeding and COVID-19: for health care workers [Internet]. Geneva: World Health Organization; 2020 May 12 [cited 2021 Aug 6]. Available from: https://apps.who.int/ iris/handle/10665/332719. [Full Text] are included in the article's Creative Commons license, unless indicated otherwise in the credit line; if the material is not included under the Creative Commons license, users will need to obtain permission from the license holder to reproduce the material. To view a copy of this license, visit http://creativecommons.org/licenses/by/4.0/ 